(n)

trobertivier Journal of Nonlinear Mathematical Physics

\title{
Symmetry reductions and new functional separable solutions of nonlinear Klein-Gordon and telegraph type equations
}

Alexei I. Zhurov, Andrei D. Polyanin

To cite this article: Alexei I. Zhurov, Andrei D. Polyanin (2020) Symmetry reductions and new functional separable solutions of nonlinear Klein-Gordon and telegraph type equations, Journal of Nonlinear Mathematical Physics 27:2, 227-242, DOI:

https://doi.org/10.1080/14029251.2020.1700633

To link to this article: https://doi.org/10.1080/14029251.2020.1700633

Published online: 04 January 2021 


\title{
Symmetry reductions and new functional separable solutions of nonlinear Klein-Gordon and telegraph type equations
}

\author{
Alexei I. Zhurov \\ Ishlinsky Institute for Problems in Mechanics, Russian Academy of Sciences, \\ 101 Vernadsky Avenue, bldg 1, 119526 Moscow, Russia; \\ Cardiff University, Heath Park, Cardiff CF14 4XY, UK \\ zhurovai@cardiff.ac.uk \\ Andrei D. Polyanin \\ Ishlinsky Institute for Problems in Mechanics, Russian Academy of Sciences, \\ 101 Vernadsky Avenue, bldg 1, 119526 Moscow, Russia; \\ Bauman Moscow State Technical University, \\ 5 Second Baumanskaya Street, 105005 Moscow, Russia; \\ National Research Nuclear University MEPhI, \\ 31 Kashirskoe Shosse, 115409 Moscow, Russia \\ polyanin@ipmnet.ru
}

Received 5 April 2019

Accepted 28 August 2019

The paper is concerned with different classes of nonlinear Klein-Gordon and telegraph type equations with variable coefficients

$$
c(x) u_{t t}+d(x) u_{t}=\left[a(x) u_{x}\right]_{x}+b(x) u_{x}+p(x) f(u),
$$

where $f(u)$ is an arbitrary function. We seek exact solutions to these equations by the direct method of symmetry reductions using the composition of functions $u=U(z)$ with $z=\varphi(x, t)$. We show that $f(u)$ and any four of the five functional coefficients $a(x), b(x), c(x), d(x)$, and $p(x)$ in such equations can be set arbitrarily, while the remaining coefficient can be expressed in terms of the others. The study investigates the properties and finds some solutions of the overdetermined system of PDEs for $\varphi(x, t)$. Examples of specific equations with new exact functional separable solutions are given. In addition, the study presents some generalized traveling wave solutions to more complex, nonlinear Klein-Gordon and telegraph type equations with delay.

Keywords: nonlinear Klein-Gordon equations; nonlinear telegraph equations; symmetry reductions; functional separable solutions; generalized traveling wave solutions; delay differential equations

2000 Mathematics Subject Classification: 81Q05, 35L70, 35J60, 35D99, 34K17

\section{Introduction}

\subsection{Brief literature review}

Nonlinear Klein-Gordon type equations play an important role in relativistic quantum mechanics, field theory, nonlinear optics, plasma physics, and particle physics. These equations describe optical fibers, ultra-short optical pulses, commensurate and incommensurate phase transitions, ferroelectric transitions, crystal growth, dislocations, and others (e.g., see $[1,9,16])$. Nonlinear telegraph type equations arise, for example, in the study of transmission lines, biological population dispersal, and random walks $[2,19]$. 
Transformations, symmetries, and exact solutions to various classes of nonlinear Klein-Gordon type equations of the form

$$
u_{t t}=\left[f_{1}(u) u_{x}\right]_{x}+f_{2}(u)
$$

have been dealt with in many studies (e.g., see $[3,5,6,15,20,23,30,31,45,53,54,63-66,71,72]$ and the literature cited therein). To obtain exact exact solutions, the authors most frequently used the classical and nonclassical symmetry methods $[3,6,31,45,54,63,64]$ and well as the methods of generalized and functional separation of variables $[5,20,23,54,72]$.

In general, equation (1.1) admits traveling wave solutions, $u=U(k x-\lambda t)$; if $f_{2}(u)=0$, it admits self-similar solutions, $u=U(x / t)$ [3]. Apart from these, equations of the form (1.1) are known to have exact solutions where the functions $f_{1}(u)$ and $f_{2}(u)$ are expressed in terms of a single arbitrary function $\varphi(u)$ [54]. It is noteworthy that if $f_{2}(u)=0$, equation (1.1) can be linearized [6,53,71]; the handbooks $[53,54]$ present a number of exact solutions in parametric form for arbitrary $f_{1}(u)$ and $f_{2}(u)=0$.

Symmetries and some exact solutions to nonlinear Klein-Gordon type equations with variable coefficients, that can be represented as the product of two functions dependent on $x$ and $u$, respectively, were considered in $[25,27,29,53,54]$. Nonlinear telegraph equations with variable coefficients of the same type were studied in $[8,26,28,53,54]$. For other more complex, related nonlinear equations of the hyperbolic type and solutions, see, for example, [4, 22, 32, 33, 39, 61].

It is also noteworthy that a number of exact solutions to nonlinear delay Klein-Gordon equations of the form

$$
u_{t t}=a u_{x x}+f(u, w), \quad w=u(x, t-\tau),
$$

where $\tau>0$ is the delay time, were obtained in [41,57].

The present paper focuses on exact solutions admitted by nonlinear Klein-Gordon and telegraph type equations of a fairly general form, including related delay equations, that involve one or more arbitrary functions. Nonlinear PDEs with arbitrary functions are most difficult to analyze symmetries and construct solutions by any methods. The most comprehensive review of the known exact solutions to such PDEs is given in [54].

It is important to note that exact solutions to nonlinear equations of mathematical physics with arbitrary functions possess significant generality and attract particular practical interest for the verification and accuracy evaluation of approximate analytical and numerical methods for the integration of related initial-boundary value problems.

\subsection{The concept of "exact solution"}

By the term "exact solution of a PDE in closed form" we understand a solution represented by one or more analytical formulas written using a predefined bounded set of allowed functions and mathematical operations. The allowed functions include the elementary functions and the functions appearing in the equation (this is required when the equation includes arbitrary functions). The allowed mathematical operations are the arithmetic operations, a finite number of function compositions, and the indefinite integral.

We will also look for exact solutions that are expressed in terms of solutions to ordinary differential equations or systems of such equations (to which the original PDE is reduced). 
Remark 1.1. In addition, for more complex, nonlinear partial differential equations with delay, exact solutions will also include those that are expressed in terms of solutions to ordinary differential equations with delay.

\section{Construction of exact solutions to nonlinear telegraph equations}

\subsection{The class of equations. Reduction of a nonlinear telegraph equation to an $\mathrm{ODE}$}

We look at nonlinear telegraph equations with variable coefficients of the form

$$
c(x) u_{t t}+d(x) u_{t}=\left[a(x) u_{x}\right]_{x}+b(x) u_{x}+p(x) f(u),
$$

where $f(u)$ is an arbitrary function. Some of the five functional coefficients $a=a(x), b=b(x)$, $c=c(x), d=d(x)$, and $p=p(x)$ can be free, while the others will be expressed in terms of them as a result of a subsequent analysis (the free coefficients can be chosen in different ways, as shown below).

The nonlinear Klein-Gordon equation is the special case of equation (2.1) with $b(x) \equiv d(x) \equiv 0$.

For $c(x) \equiv 0$, equation (2.1) degenerates into a nonlinear convection-diffusion equation with volume reaction. For symmetry reductions and exact solutions to similar and related nonlinear equations of the parabolic type, see, for example, $[10-12,17,18,21,24,34-36,38,42,47-50,53-56,58-$ 60, 67-69].

We seek exact solutions to equation (2.1) using the direct method of symmetry reductions [13, $14]$ in the form of a composition of functions $[48,49]$ :

$$
u=U(z), \quad z=\varphi(x, t) .
$$

Substituting (2.2) into (2.1) yields the equation

$$
\left[a(x) \varphi_{x}^{2}-c(x) \varphi_{t}^{2}\right] U_{z z}^{\prime \prime}+\left\{\left[a(x) \varphi_{x}\right]_{x}-c(x) \varphi_{t t}+b(x) \varphi_{x}-d(x) \varphi_{t}\right\} U_{z}^{\prime}+p(x) f(U)=0 .
$$

In the special case $U(z)=z$, equation (2.3) coincides with the original equation (2.1), which means that no solution has been lost at this stage. Now let us require that the relations

$$
\begin{aligned}
p(x) & =s(\varphi)\left[a(x) \varphi_{x}^{2}-c(x) \varphi_{t}^{2}\right], \\
c(x) \varphi_{t t}+d(x) \varphi_{t} & =\left[a(x) \varphi_{x}\right]_{x}+b(x) \varphi_{x}+k(\varphi)\left[a(x) \varphi_{x}^{2}-c(x) \varphi_{t}^{2}\right]
\end{aligned}
$$

hold, where $s(\varphi)$ and $k(\varphi)$ are some functions $(s \not \equiv 0)$. Then the variables separate and equation (2.3) reduces to the ordinary differential equation

$$
U_{z z}^{\prime \prime}-k(z) U_{z}^{\prime}+s(z) f(U)=0
$$

For exact solutions to the nonlinear ordinary differential equation (2.6) with some specific functions $k(z), s(z)$, and $f(U)$, see [52].

In the special case $k(z) \equiv 0$, in which equation (2.5) become linear, the general solution to equation (2.6) with $s(z)=1$ and any $f(U)$ can be represented in implicit form:

$$
\int\left[C_{1}-2 \int f(U) d U\right]^{-1 / 2} d U=C_{2} \pm z
$$

where $C_{1}$ and $C_{2}$ are arbitrary constants. 
Equations (2.4)-(2.6) allow one to effectively find exact solutions to a wide class of nonlinear telegraph equations of the form (2.1).

Remark 2.1. In equation (2.1), two out of the five functional coefficients $a(x), b(x), c(x), d(x)$, and $p(x)$ can be set equal to unity without loss of generality. In particular, dividing the equation by $c$ and changing from $t$ and $x$ to the new variables $t$ and $y=\int \sqrt{c / a} d x$, we arrive at the equation in a canonical form $u_{t t}+d_{1}(y) u_{t}=u_{y y}+b_{1}(y) u_{y}+p_{1}(y) f(u)$. It is not difficult to find a transformation $t, \bar{y}=\bar{y}(x)$ that would reduce equation (2.1) to another canonical form: $u_{t t}+d_{2}(\bar{y}) u_{t}=\left[a_{2}(\bar{y}) u_{\bar{y}}\right]_{\bar{y}}+$ $p_{2}(\bar{y}) f(u)$. However, analyzing the equation in the general form (2.1) is more convenient, since it contains all its canonical and non-canonical forms.

Remark 2.2. Denoting $a(x)=A(x)$ and $b(x)=B(x)-a_{x}^{\prime}(x)$, we can rewrite equation (2.1) in the alternative form $c(x) u_{t t}+d(x) u_{t}=A(x) u_{x x}+B(x) u_{x}+p(x) f(u)$.

Remark 2.3. In equations (2.1) and (2.3)-(2.5), the functions $a(x), b(x), c(x), d(x)$, and $p(x)$ can be replaced with functions of two arguments: $a(x, t), b(x, t), c(x, t), d(x, t)$, and $p(x, t)$. All reasoning will remain the same.

\subsection{The determining system of equations and its properties. The direct procedure for seeking exact solutions}

In the generic case, equations (2.4) and (2.5) with prescribed functions $a=a(x), b=b(x), c=c(x)$, $d=d(x), p=p(x), k(\varphi)$, and $s(\varphi)$ make up an overdetermined nonlinear system of equations for $\varphi$. This system will be referred to as the determining system of equations.

Nonlinear transformations

$$
\varphi=F(\psi)
$$

preserve the form of equations (2.4) and (2.5), while the functional coefficients $k(\varphi)$ and $s(\varphi)$ will change by the rule

$$
k(\varphi) \Longrightarrow k(F(\psi)) F_{\psi}^{\prime}(\psi)+\frac{F_{\psi \psi}^{\prime \prime}(\psi)}{F_{\psi}^{\prime}(\psi)}, \quad s(\varphi) \Longrightarrow s(F(\psi))\left[F_{\psi}^{\prime}(\psi)\right]^{2} .
$$

The degenerate case $k(\varphi) \equiv 0$ corresponds to the linear hyperbolic equation with variable coefficients (2.5). If $k(\varphi) \not \equiv 0$, the substitution

$$
\psi=C_{1} \int K(\varphi) d \varphi+C_{2}, \quad K(\varphi)=\exp \left[\int k(\varphi) d \varphi\right]
$$

where $C_{1}$ and $C_{2}$ are arbitrary constants, linearizes equation (2.5) to give

$$
c(x) \psi_{t t}+d(x) \psi_{t}=\left[a(x) \psi_{x}\right]_{x}+b(x) \psi_{x} .
$$

In the special case $k(\psi)=k=$ const, one can use the substitution

$$
\varphi=k^{-1} \ln |\psi|,
$$

which follows from (2.10).

Since transformations of the form (2.8) only change the functional coefficients $k(\varphi)$ and $s(\varphi)$ in equations (2.4) and (2.5), the function $F$ can be chosen, without loss of generality, so as to simplify one of these equations. 
The direct procedure for constructing exact solutions to nonlinear equations of the form (2.1) assumes the functions $a(x), b(x), c(x), d(x)$, and $f(u)$ to be given while the functions $u=u(x)$ and $p=p(x)$ to be unknown. Then, by setting $k(\varphi)$ and $s(\varphi)$ somehow, one needs first to find particular solitons $p(x)$ and $\varphi=\varphi(x, t)$ to equations (2.4) and (2.5); recall that the latter equation can be linearized, as shown above. After that, taking into account (2.4), one obtains the corresponding solution to equation (2.1) by formula (2.2), with the function $U(z)$ being a solution to the ordinary differential equation (2.6).

Solutions to the linear equations (2.5), with $k(\varphi) \equiv 0$, and (2.11) can be obtained using the method of separation of variables. In particular, equation (2.11) with $d(x) \equiv 0$ admits the exact solutions

$$
\begin{array}{lll}
\psi=\alpha t^{2}+\beta t+\zeta(x), & & {\left[a(x) \zeta_{x}^{\prime}\right]_{x}^{\prime}+b(x) \zeta_{x}^{\prime}-2 \alpha c(x)=0 ;} \\
\psi=[\alpha \exp (-\lambda t)+\beta \exp (\lambda t)] \zeta(x), & & {\left[a(x) \zeta_{x}^{\prime}\right]_{x}^{\prime}+b(x) \zeta_{x}^{\prime}-\lambda^{2} c(x) \zeta=0,} \\
\psi=[\alpha \cos (\lambda t)+\beta \sin (\lambda t)] \zeta(x), & & {\left[a(x) \zeta_{x}^{\prime}\right]_{x}^{\prime}+b(x) \zeta_{x}^{\prime}+\lambda^{2} c(x) \zeta=0,}
\end{array}
$$

where $\alpha, \beta, \lambda$ are arbitrary constants. The ODE in (2.13) is easy to integrate with the substitution $w(x)=\zeta_{x}$. A number of solutions to the linear equations of (2.14) and (2.15) with various specific $a(x), b(x)$, and $c(x)$ can be found in [52]. For other exact solutions to equations (2.11) with specific $a(x), b(x)$, and $c(x)$, see [51].

In subsequent sections, without pretending to an exhaustive analysis of the overdetermined system (2.4), (2.5), we will show how this system allows us to construct exact solutions to equations of the form (2.1), once suitable functional coefficients $k(\varphi)$ and $s(\varphi)$ are selected.

\subsection{The case of $k(\varphi)=k$ and $s(\varphi)=1$. Generalized traveling wave solutions}

The nonlinear telegraph equations (2.1) admit generalized traveling wave solutions (2.2) with

$$
\varphi(x, t)=t+\int g(x) d x .
$$

The function $g(x)$ can be either preset or determined in a subsequent analysis, depending on the goal (see below). Substituting (2.16) into (2.4) and (2.5) followed by setting $s(\varphi)=1$ and $k(\varphi)=$ $k=$ const, we obtain

$$
\begin{aligned}
& p(x)=a(x) g^{2}(x)-c(x), \\
& d(x)=[a(x) g(x)]_{x}^{\prime}+b(x) g(x)+k\left[a(x) g^{2}(x)-c(x)\right] .
\end{aligned}
$$

Equation (2.18) relates the first four functional coefficients of equations (2.1) and the function $g=g(x)$ appearing in (2.16); it is a differential relation with respect to $a$ and $g$ and an algebraic relation with respect to $b, c$ and $d$. Equation (2.17) is algebraic; it serves to determine the functional coefficient $p(x)$.

If the four functions $a(x), b(x), c(x)$, and $d(x)$ are assumed to be given, relation (2.18) with $k \neq 0$ represents a Riccati equation for $g=g(x)$, which can be written as

$$
a(x) g_{x}^{\prime}+k a(x) g^{2}+\left[b(x)+a_{x}^{\prime}(x)\right] g-k c(x)-d(x)=0 .
$$

A wide list of exact solutions to this equation for various specific $a(x), b(x), c(x)$, and $d(x)$ can be found in [52]. Below we will look at two cases. 
Degenerate case. If $k=0$, the Riccati equation (2.19) degenerates into a linear equation, whose general solution is

$$
g(x)=\frac{E(x)}{a(x)}\left[\int \frac{d(x)}{E(x)} d x+m\right], \quad E(x)=\exp \left[-\int \frac{b(x)}{a(x)} d x\right]
$$

where $m$ is an arbitrary constant.

Example 2.1. Suppose that one of the equation coefficients is arbitrarily dependent on the space variable, $a=a(x)$, while the other three are constant, $b(x)=d(x)=0$ and $c(x)=1$. By formula (2.20) we get $g(x)=m / a(x)$. Inserting this into (2.16) and (2.17), we find that $\varphi(x)=$ $t+m \int \frac{d x}{a(x)}$ and $p(x)=\frac{m^{2}}{a(x)}-1$. Hence, the nonlinear Klein-Gordon type equation

$$
u_{t t}=\left[a(x) u_{x}\right]_{x}+\left[\frac{m^{2}}{a(x)}-1\right] f(u),
$$

which involves two arbitrary functions, $a(x)$ and $f(u)$, admits the functional separable solution

$$
u=U(z), \quad z=t+m \int \frac{d x}{a(x)},
$$

with the function $U(z)$ determined by the solvable autonomous ordinary differential equation

$$
U_{z z}^{\prime \prime}+f(U)=0 .
$$

This equation is obtained by substituting $k=0$ and $s=1$ in (2.6); its general solution can be represented in the implicit form (2.7).

For instance, by setting $a(x)=e^{\lambda x}$ in (2.21), we get the nonlinear equation

$$
u_{t t}=\left(e^{\lambda x} u_{x}\right)_{x}+\left(m^{2} e^{-\lambda x}-1\right) f(u),
$$

which admits a generalized traveling wave solution for arbitrary $f(u)$.

Example 2.2. Let us look at the case of $a=a(x), b(x)=0$, and $c(x)=d(x)=1$. By formulas (2.20) with $m=0$, we get $g(x)=x / a(x)$. Substituting this into (2.16) and (2.17) gives $\varphi(x)=t+\int \frac{x d x}{a(x)}$ and $p(x)=\frac{x^{2}}{a(x)}-1$. It follows that the nonlinear telegraph equation

$$
u_{t t}+u_{t}=\left[a(x) u_{x}\right]_{x}+\left[\frac{x^{2}}{a(x)}-1\right] f(u)
$$

involving two arbitrary functions, $a(x)$ and $f(u)$, admits the functional separable solution

$$
u=U(z), \quad z=t+\int \frac{x d x}{a(x)}
$$

where $U(z)$ is a function determined by the solvable differential equation (2.23).

Example 2.3. Now we set $a=a(x), b=-a_{x}(x), c(x)=1$, and $d(x)=0$ and use formulas (2.20) to get $g(x)=m$. Substituting into (2.16) and (2.17) gives $\varphi(x)=t+m x$ and $p(x)=m^{2} a(x)-1$. It 
follows that the Klein-Gordon type equation

$$
u_{t t}=a(x) u_{x x}+\left[m^{2} a(x)-1\right] f(u),
$$

which involves two arbitrary functions, $a(x)$ and $f(u)$, admits the exact solution

$$
u=U(z), \quad z=t+m x,
$$

with the function $U(z)$ described by the solvable autonomous ordinary differential equation (2.23).

Remark 2.4. Solution (2.28) is a noninvariant traveling wave solution to equation (2.27), which means that it cannot be obtained using the group analysis of the equation. The function $a(x)$ in equation (2.27) can be replaced with $a(x, t)$.

Nondegenerate case. If $k=$ const $(k \neq 0)$, the change of variable

$$
g=\frac{1}{k} \frac{y_{x}^{\prime}}{y}
$$

converts (2.19) to a second-order linear differential equation

$$
a(x) y_{x x}^{\prime \prime}+\left[b(x)+a_{x}^{\prime}(x)\right] y_{x}^{\prime}-k[k c(x)+d(x)] y=0 .
$$

An extensive list of exact solutions to this equation for various specific $a(x), b(x), c(x)$, and $d(x)$ can be found in [52].

Example 2.4. In the case of $a=c=1$ and $b=d=0$, the general solution of equation (2.30) is

$$
y=C_{1} \cosh (k x)+C_{2} \sinh (k x),
$$

where $C_{1}$ and $C_{2}$ are arbitrary constants. By setting $C_{1}=1, C_{2}=0$, and $k=1$ in (2.31), we find using formula (2.29) that

$$
g(x)=\tanh x .
$$

Inserting this into (2.16) and (2.17), we get

$$
\varphi(x)=t+\ln \cosh x, \quad p(x)=-1 / \cosh ^{2} x .
$$

It follows that the nonlinear Klein-Gordon type equation

$$
u_{t t}=u_{x x}-\frac{1}{\cosh ^{2} x} f(u)
$$

with arbitrary $f(u)$ admits the functional separable solution

$$
u=U(z), \quad z=t+\ln \cosh x
$$

where $U(z)$ is a function satisfying the autonomous ordinary differential equation

$$
U_{z z}^{\prime \prime}-U_{z}^{\prime}+f(U)=0 .
$$

The order of equation (2.34) can be reduced by one with the substitution $U_{z}^{\prime}=\Phi(U)$, which leads to an Abel equation of the second kind in the canonic form. For exact solutions to equation (2.34) with certain specific $f(U)$, see [52]. 
Other ways of constructing exact solutions. We now discuss other possibilities to construct exact solutions to equations of the form (2.1) with $k(\varphi)=k$ and $s(\varphi)=1$ without integrating the Riccati equation (2.19). To this end, we preset the function $g(x)$ as well as any three out of the four functions $a(x), b(x), c(x)$, and $d(x)$ (treat these functions to be free), while the remaining function will be expressed in terms of them using (2.19). Table 1 lists possible situations and gives formulas for the function expressed via the others. The form of the nonlinear telegraph equation is determined by the substitution of $p(x)=a(x) g^{2}(x)-c(x)$ in $(2.1)$.

Table 1. Different ways of setting the functional coefficients in (2.1) with $p(x)=a(x) g^{2}(x)-c(x)$.

No Preset free functions

Function expressed via the free ones

$$
\begin{array}{lll}
1 & a=a(x), b=b(x), d=d(x), g=g(x) & c(x)=k^{-1}\left[a g_{x}^{\prime}+k a g^{2}+\left(b+a_{x}^{\prime}\right) g-d\right] \\
2 & a=a(x), c=c(x), d=d(x), g=g(x) & b(x)=g^{-1}\left(k c+d-a g_{x}^{\prime}\right)-a_{x}^{\prime}-k a g \\
3 & a=a(x), b=b(x), c=c(x), g=g(x) & d(x)=a g_{x}^{\prime}+k a g^{2}+\left(b+a_{x}^{\prime}\right) g-k c \\
4 & b=b(x), c=c(x), d=d(x), g=g(x) & a(x)=g^{-1} E\left[\int(k c+d-b g) E^{-1} d x+C_{1}\right]
\end{array}
$$

Note: $k$ and $C_{1}$ are arbitrary constants, $g^{-1}=1 / g$, and $E=\exp \left(-k \int g d x\right)$.

Example 2.5. For an alternative representation of exact solutions to the equation in question, we use the fourth way of setting the functional coefficients (see Table 1) with $b=d=0$ and $c=1$. Two cases are possible.

1. Degenerate case, $k=0$. From row 4 of Table 1 , we get $a(x)=C_{1} g^{-1}(x)$ and $p(x)=C_{1} g(x)-1$. Up to notation, this leads to equation (2.21) and its solution (2.22).

2. Nondegenerate case, $k \neq 0$. From row 4 of Table 1 with $k \neq 0$ and $C_{1}=0$, we get $a(x)=$ $\mathrm{kg}^{-1} E \int E^{-1} d x$. Introducing the function $h(x)=\int E^{-1} d x$, computing its derivative, and taking into account that $E=\exp \left(-k \int g d x\right)$, we express $g$ in terms of $h$. We find after simple rearrangements that $g=k^{-1} h_{x x}^{\prime \prime} / h_{x}^{\prime}, a=k^{2} h / h_{x x}^{\prime \prime}$, and $p=h\left(h_{x}^{\prime}\right)^{-2} h_{x x}^{\prime \prime}-1$. It follows that the equation

$$
u_{t t}=\left[a(x) u_{x}\right]_{x}+p(x) f(u), \quad a(x)=k^{2} \frac{h}{h_{x x}^{\prime \prime}}, \quad p(x)=\frac{h h_{x x}^{\prime \prime}}{\left(h_{x}^{\prime}\right)^{2}}-1,
$$

where $f(u)$ and $h=h(x)$ are arbitrary functions and $k \neq 0$ is an arbitrary constant, admits the generalized functional separable solution

$$
u=U(z), \quad z=t+\frac{1}{k} \ln \left|h_{x}^{\prime}\right| .
$$

The function $U(z)$ is determined by the ordinary differential equation $U_{z z}^{\prime \prime}-k U_{z}^{\prime}+f(U)=0$.

By setting, for instance, $h=\sinh x$ and $k=1$ in (2.35), we obtain equation (2.32) and its exact solution (2.33). 


\subsection{The case of $d(x)=0, k(\varphi)=k_{0} / \varphi$, and $s(\varphi)=s_{0} / \varphi$. Generalized separable solutions}

If $d(x)=0, k(\varphi)=k_{0} / \varphi$, and $s(\varphi)=s_{0} / \varphi$, the overdetermined system (2.4)-(2.5) admits solutions of the form

$$
\varphi(x, t)=\theta(x)-\left(t+t_{0}\right)^{2},
$$

where $t_{0}$ is an arbitrary constant. The function $\theta=\theta(x)$ is determined by the ordinary differential equation

$$
a(x)\left(\theta_{x}^{\prime}\right)^{2}=4 c(x) \theta
$$

Equations (2.4) and (2.5) then reduce to

$$
\begin{aligned}
& p(x)=4 s_{0} c(x), \\
& {\left[a(x) \theta_{x}^{\prime}\right]_{x}^{\prime}+b(x) \theta_{x}^{\prime}+\left(4 k_{0}+2\right) c(x)=0 .}
\end{aligned}
$$

Let us look at the special case $b(x)=0$ in more detail. We assume the function $c=c(x)$ to be given. Then the function $p(x)$ is determined by formula (2.38), while $a=a(x)$ and $\theta=\theta(x)$ are found from equations (2.37) and (2.39). Omitting the intermediate calculations, we obtain if $k_{1}=4 k_{0}+2 \neq 0$,

$$
a(x)=\frac{1}{4 C_{1} c(x)} I^{\frac{4}{k_{1}}+2}, \quad \theta=C_{1} I^{-\frac{4}{k_{1}}}, \quad I=C_{2}-k_{1} \int c(x) d x
$$

if $k_{0}=-\frac{1}{2}$ :

$$
a(x)=\frac{C_{1}^{2}}{4 C_{2} c(x) E(x)}, \quad \theta=C_{2} E(x), \quad E(x)=\exp \left[\frac{4}{C_{1}} \int c(x) d x\right],
$$

where $C_{1}$ and $C_{2}$ are arbitrary constants.

Example 2.6. With $b(x)=0, c(x)=p(x)=1, s_{0}=\frac{1}{4}$, and $k_{0}=-\frac{1}{2}$, relation (2.38) is satisfied identically, while formulas (2.41) become

$$
a(x)=\frac{C_{1}^{2}}{4 C_{2}} \exp \left(-\frac{4}{C_{1}} x\right), \quad \theta(x)=C_{2} \exp \left(\frac{4}{C_{1}} x\right) .
$$

Setting $C_{1}=-4 / \lambda$ and $C_{2}=4 / \lambda^{2}$ in (2.42), we arrive at the nonlinear Klein-Gordon type equation

$$
u_{t t}=\left(e^{\lambda x} u_{x}\right)_{x}+f(u) .
$$

For arbitrary $f(u)$, it admits a functional separable solution of the form

$$
u=U(z), \quad z=4 \lambda^{-2} e^{-\lambda x}-t^{2},
$$

where $U(z)$ is a function satisfying the nonautonomous ordinary differential equation

$$
4 z U_{z z}^{\prime \prime}+2 U_{z}^{\prime}+f(U)=0 .
$$

Remark 2.5. Solution (2.44) to equation (2.43) was obtained in [53]. 
Solution 7. Now we set $a(x)=c(x)=p(x)=1$ and $s_{0}=\frac{1}{4}$, in which case equation (2.38) is satisfied identically, while solutions to equations (2.37) and (2.39) are given by

$$
b(x)=-\frac{2 k_{0}}{x+C_{1}}, \quad \theta(x)=\left(x+C_{1}\right)^{2},
$$

where $C_{1}$ is an arbitrary constant. To $C_{1}=0$ and $k_{0}=-\frac{1}{2}(n-1)$ there corresponds an $n$-dimensional nonlinear Klein-Gordon equation with radial symmetry [53]:

$$
u_{t t}=u_{x x}+\frac{n-1}{x} u_{x}+f(u) \text {. }
$$

It admits a solution of the form $u=U(z)$ with $z=x^{2}-\left(t+t_{0}\right)^{2}$ and $x$ being the radial coordinate.

\subsection{The case $d(x)=0$. Solutions to the determining system in the multiplicative form $\varphi=\xi(x) t$}

We look for compatible solutions to the determining system (2.4)-(2.5) in the form

$$
\varphi=\xi(x) t .
$$

A simple analysis shows that solution (2.46) satisfies both equation (2.4) and (2.5) if and only if the following conditions hold:

$$
p=s_{0} c \xi^{2}, \quad k(\varphi)=\frac{k_{0} \varphi}{A^{2} \varphi^{2}-1}, \quad s(\varphi)=\frac{s_{0}}{A^{2} \varphi^{2}-1},
$$

where $A, k_{0}$, and $s_{0}$ are some constants (which can be varied). The functions $a=a(x), b=b(x)$, $c=c(x)$, and $\xi=\xi(x)$ are connected by two differential-algebraic relations

$$
a\left(\xi_{x}^{\prime}\right)^{2}=A^{2} c \xi^{4}, \quad\left(a \xi_{x}^{\prime}\right)_{x}^{\prime}+b \xi_{x}^{\prime}+k_{0} c \xi^{3}=0 .
$$

Any two of the four functions in (2.48) can be treated as given (arbitrarily) and the other two as unknown.

Let us look at the special case of $b(x)=0$ and $c(x)=1$. Eliminating $a$ from (2.48), we arrive at the following differential equation for $\xi$ :

$$
\xi \xi_{x x}^{\prime \prime}=\left(k_{0} A^{-2}+4\right)\left(\xi_{x}^{\prime}\right)^{2} .
$$

Equation (2.49) is autonomous and generalized homogeneous. Its general solution is

$$
\xi= \begin{cases}C_{1}\left(x+C_{2}\right)^{-\frac{A^{2}}{k_{0}+3 A^{2}}} & \text { if } k_{0} \neq-3 A^{2}, \\ C_{1} e^{\lambda x} & \text { if } k_{0}=-3 A^{2},\end{cases}
$$

where $C_{1}, C_{2}$, and $\lambda$ are arbitrary constants. The function $a$ is expressed via $\xi$ as $a=A^{2} \xi^{4}\left(\xi_{x}^{\prime}\right)^{-2}$, which follows from the first equation of (2.48).

Example 2.7. By setting $A=C_{1}=s_{0}=1, k_{0}=-3, \lambda=1 / 2, b(x)=0$, and $c(x)=1$ in (2.47) and (2.50), we get $\xi(x)=e^{x / 2}$ and $a(x)=p(x)=e^{x}$. It follows that the nonlinear Klein-Gordon type equation

$$
u_{t t}=\left(e^{x} u_{x}\right)_{x}+e^{x} f(u)
$$

with arbitrary $f(u)$ admits an exact invariant solution of the form $u=U(z)$ with $z=e^{x / 2} t$. 


\subsection{Relationship of the direct method of symmetry reductions with other methods}

1. Relationship with the method of differential constraints. The direct method of symmetry reductions can be reformulated in terms of the method of differential constraints [70] (this was proved in [44] for the general case), which is based on the compatibility theory of PDEs. To show this, we differentiate formula (2.2), for example, with respect to $t$ to obtain the differential relation

$$
u_{t}=\eta(x, t) \Psi(u)
$$

where $\eta(x, t)=\varphi_{t}(x, t)$ and the function $\Psi(u)$ is defined parametrically by the relations $u=U(z)$ and $\Psi=U_{z}^{\prime}(z)$.

Relation (2.51) can be treated as a first-order differential constraint, which can be used to find exact solutions of equation (2.1) through a compatibility analysis of the overdetermined pair of equations (2.1) and (2.51) with the single unknown $u$. At the initial stage, all functions included on the right-hand sides of (2.51) are considered arbitrary, and the specific form of these functions is determined in the subsequent analysis. In the general case, any PDE that depends on the same variables as the original equation can be treated as a differential constraint.

For a description of the method of differential constraints and its relationship with other methods as well as a number of specific examples of its application, see, for example, [5, 21, 37, 44, 54, 70].

It is important to note that the construction of exact solutions by the method of differential constraints is based on a compatibility analysis of PDEs and is carried out in several steps; in three of the steps, one has to solve nonlinear differential equations (e.g., see [49,54] for details). Examples of using differential constraints of the form (2.51) can be found in [46].

2. Relationship with the nonclassical method of symmetry reductions. The first-order differential constraint (2.51) is a special case of the invariant surface condition [7], which characterizes the nonclassical method of symmetry reduction. (In general, an invariant surface condition is a quasilinear first-order PDE of general form.) This method, just like the method of different constraints, also relies on a compatibility analysis of two PDEs; specific examples of its use can be found, for example, in $[7,14,40,43,54,62]$. For first-order differential constraints, the results of applying the method of differential constraints and the nonclassical method of symmetry reduction coincide (provided that the differential constraint coincides with the invariant surface condition).

In practice, it is technically much easier to use the direct method of symmetry reduction, which requires fewer steps where intermediate differential equations must be solved with the intermediate equations being simpler, than the method of differential constraints or the nonclassical method of symmetry reductions. In all cases, a complicating factor is the presence of arbitrary functions if included in the equation in question; it is precisely such equations that are dealt with in the present study.

\section{Exact solutions to nonlinear delay telegraph equations}

\subsection{Nonlinear delay equations admitting generalized traveling wave solutions}

The results obtained in Section 2 can be extended to the case of more complex, nonlinear delay telegraph equations of the form

$$
c(x) u_{t t}+d(x) u_{t}=\left[a(x) u_{x}\right]_{x}+b(x) u_{x}+p(x) f(u, w), \quad w=u(x, t-\tau),
$$


where $\tau$ is a constant representing the delay time and $f(u, w)$ is an arbitrary function of two arguments.

Below we show how solutions to the nonlinear telegraph equation without delay (2.1), which are defined by formulas (2.2) and (2.16), can be used to construct exact solutions to the nonlinear telegraph equation with delay (3.1). Suppose equation (2.1) admits a generalized traveling wave solution of the form

$$
u=U(z), \quad z=t+\theta(x)
$$

with the function $U(z)$ satisfying the ordinary differential equation (2.6). Then the delay equation (3.1) admits solution (3.2) with $U(z)$ satisfying the delay ordinary differential equation

$$
U_{z z}^{\prime \prime}-k(z) U_{z}^{\prime}+s(z) f(U, W)=0, \quad W=U(z-\tau) .
$$

Equations (2.21), (2.24), (2.25), (2.27), (2.32), and (2.35) admit solutions of the form (3.2). Therefore, such solutions are also admitted by the more complex nonlinear telegraph equations that are obtained from the above ones by replacing $f(u)$ with $f(u, w)$.

Example 3.1. The nonlinear Klein-Gordon type equation with delay

$$
u_{t t}=\left[a(x) u_{x}\right]_{x}+\left[\frac{m^{2}}{a(x)}-1\right] f(u, w), \quad w=u(x, t-\tau),
$$

which is a generalization of equation (2.21), admits, for arbitrary $a(x)$ and $f(u, w)$, the generalized traveling wave solution

$$
u=U(z), \quad z=t+m \int \frac{d x}{a(x)},
$$

with the function $U(z)$ described by the autonomous ordinary differential equation with delay

$$
U_{z z}^{\prime \prime}+f(U, W)=0, \quad W=U(z-\tau) .
$$

Note that if $f(U, W)=U g(W / U)$, equation (3.6) admits a solution of the form $U=C e^{\lambda z}$, where $C$ is an arbitrary constant and $\lambda$ is a root of the transcendental equation $\lambda^{2}+g\left(e^{-\tau \lambda}\right)=0$.

Example 3.2. The nonlinear telegraph equation with delay

$$
u_{t t}+u_{t}=\left[a(x) u_{x}\right]_{x}+\left[\frac{x^{2}}{a(x)}-1\right] f(u, w), \quad w=u(x, t-\tau),
$$

which is a generalization of equation (2.25), admits, for arbitrary $a(x)$ and $f(u, w)$, the generalized traveling wave solution

$$
u=U(z), \quad z=t+\int \frac{x d x}{a(x)}
$$

with the function $U(z)$ described by the ordinary differential equation with delay (3.6). 


\subsection{Nonlinear delay equations that admit multiplicative separable solutions}

The nonlinear telegraph equations with delay

$$
c(x) u_{t t}+d(x) u_{t}=\left[a(x) u_{x}\right]_{x}+b(x) u_{x}+u F(x, w / u), \quad w=u(x, t-\tau),
$$

which involves five arbitrary functions $a(x), b(x), c(x), d(x)$, and $F(x, v)$, admits an exact solution in the multiplicative form $u=e^{\lambda t} \xi(x)$, where $\lambda$ is an arbitrary constant and $\xi=\xi(x)$ is a function satisfying the linear ordinary differential equation

$$
\left[a(x) \xi_{x}^{\prime}\right]_{x}^{\prime}+b(x) \xi_{x}^{\prime}+\left[F\left(x, e^{-\lambda \tau}\right)-\lambda^{2} c(x)-\lambda d(x)\right] \xi=0 .
$$

Some exact solutions to these equations can be found in [52].

In the special case $d(x)=0$, equation (3.7) also admits exact solutions of the form

$$
u=\sin \left(\beta_{n} t+C\right) \xi(x),
$$

where $\beta_{n}=\pi n / \tau(n=1,2, \ldots)$ and $C$ is an arbitrary constant.

\section{Brief conclusions}

We have described several classes of nonlinear Klein-Gordon and telegraph type equations with variable coefficients that admit functional separable solutions. The focus was on fairly general equations that involve one or more arbitrary functions. A number of specific equations with solutions have been presented. A few generalized traveling wave solutions have been obtained for more complex, nonlinear telegraph equations with delay.

\section{Acknowledgments}

The research was supported by the Ministry of Education and Science of the Russian Federation within the framework of the State Assignment (registration Nos. AAAA-A17-117021310385-6 and AAAA-A17-117021310385-7) and partially supported by the Russian Foundation for Basic Research (project No. 18-29-10025).

\section{References}

[1] R. Abazari and S. Jamshidzadeh, Exact solitary wave solutions of the complex Klein-Gordon equation, Optik, 126 (2015), 1970-1975.

[2] E. Ahmed, H.A. Abdusalam and E.S. Fahmy, On telegraph reaction diffusion and coupled maplattice in some biological systems, Int. J. Modern Phys. C, 12 (2001), 717-726.

[3] W.F. Ames, J.R. Lohner and E. Adams, Group properties of $u_{t t}=\left[f(u) u_{x}\right]_{x}$. Int. J. Non-Linear Mech., 16 (5-6) (1981), 439-447.

[4] S.C. Anco and S. Liu, Exact solutions of semilinear radial wave equations in $n$ dimensions, J. Math. Anal. Appl., 297 (2004), 317-342.

[5] V.K. Andreev, O.V. Kaptsov, V.V. Pukhnachov and A.A. Rodionov, Applications of Group-Theoretical Methods in Hydrodynamics (Dordrecht, Kluwer, 1998).

[6] G.W. Bluman and A.F. Cheviakov, Nonlocally related systems, linearization and nonlocal symmetries for the nonlinear wave equation, J. Math. Anal. Appl., 333 (2007), 93-111.

[7] G.W. Bluman and J.D. Cole, The general similarity solution of the heat equation, J. Math. Mech., 18 (1969), 1025-1042.

[8] G.W. Bluman, Temuerchaolu and R. Sahadevan, Local and nonlocal symmetries for nonlinear telegraph equation, J. Math. Phys., 46 (2005), 023505. 
[9] B. Bulbul, M. Sezer and W. Greiner, Relativistic Quantum Mechanics-Wave Equations, 3rd edition (Berlin, Springer, 2000).

[10] R.M. Cherniha and O. Pliukhin, New conditional symmetries and exact solutions of nonlinear reactiondiffusion-convection equations, J. Physics A: Math. Theor., 40 (33) (2007), 10049-10070.

[11] R.M. Cherniha and O. Pliukhin, New conditional symmetries and exact solutions of reaction-diffusionconvection equations with exponential nonlinearities, J. Math. Anal. Appl., 403 (2013), 23-37.

[12] R. Cherniha, M. Serov and O. Pliukhin, Nonlinear Reaction-Diffusion-Convection Equations: Lie and Conditional Symmetry, Exact Solutions and Their Applications (Boca Raton, Chapman \& Hall/CRC Press, 2018).

[13] P.A. Clarkson and M.D. Kruskal, New similarity reductions of the Boussinesq equation, J. Math. Phys., 30 (1989), 2201-2213.

[14] P.A. Clarkson, D.K. Ludlow and T.J. Priestley, The classical, direct and nonclassical methods for symmetry reductions of nonlinear partial differential equations, Methods Appl. Anal., 4 (2) (1997), 173-195.

[15] P.A. Clarkson, J.B. McLeod, P.J. Olver and R. Ramani, Integrability of Klein-Gordon equations, SIAM J. Math. Anal., 17 (1986), 798-802.

[16] J. Cuevas-Maraver et al. (eds.), The sine-Gordon Model and its Applications, (Heidelberg, Springer, 2014).

[17] V.A. Dorodnitsyn, On invariant solutions of the equation of non-linear heat conduction with a source, USSR Comput. Math. \& Math. Phys., 22 (6) (1982), 115-122.

[18] P.W. Doyle and P.J. Vassiliou, Separation of variables for the 1-dimensional non-linear diffusion equation, Int. J. Non-Linear Mech., 33 (2) (1998), 315-326.

[19] S.R. Dunbar and H.G. Othmer, On a nonlinear hyperbolic equation describing transmission lines, cell movement, and branchingrandom walks, In: H.G. Othmer (ed.), Nonlinear Oscillations in Biology and Chemistry, in: Lecture Notes in Biomath., 66 274-289 (Berlin, Springer-Verlag, 1986).

[20] P.G. Estévez and C.Z. Qu, Separation of variables in nonlinear wave equations with variable wave speed, Theor. Math. Phys., 133 (2) (2002), 1490-1497.

[21] V.A. Galaktionov, Quasilinear heat equations with first-order sign-invariants and new explicit solutions, Nonlinear Anal.: Theory, Methods \& Appl., 23 (1994), 1595-1621.

[22] M.L. Gandarias, M. Torrisi and A. Valenti, Symmetry classification and optimal systems of a non-linear wave equation, Int. J. Non-Linear Mech., 39 (2004), 389-398.

[23] A.M. Grundland and E. Infeld, A family of non-linear Klein-Gordon equations and their solutions, J. Math. Phys., 33 (1992), 2498-2503.

[24] S. Hood, On direct, implicit reductions of a nonlinear diffusion equation with an arbitrary function generalizations of Clarkson's and Kruskal's method, IMA J. Appl. Math., 64 (3) (2000), 223-244.

[25] J. Hu and C. Qu, Functionally separable solutions to nonlinear wave equations by group foliation method, J. Math. Anal. Appl., 330 (2007), 298-311.

[26] D.J. Huang and N.M. Ivanova, Group analysis and exact solutions of a class of variable coefficient nonlinear telegraph equations, J. Math. Phys., 48 (2007), 073507.

[27] D.J. Huang and S. Zhou, Group properties of generalized quasi-linear wave equations. J. Math. Anal. Appl., 366 (2010), 460-472.

[28] D.J. Huang and S. Zhou, Group-theoretical analysis of variable coefficient nonlinear telegraph equations. Acta Appl. Math., 117 (1) (2012), 135-183.

[29] D.J. Huang, Y. Zhu and Q. Yang, Reduction operators and exact solutions of variable coefficient nonlinear wave equations with power nonlinearities, Symmetry, 7 (1) (2017), 3.

[30] G. Hwang, The elliptic sinh-Gordon equation in the quarter plane, J. Nonlinear Math. Phys., 23 (1) (2016),127-140.

[31] N.H. Ibragimov (ed.), CRC Handbook of Lie Group Analysis of Differential Equations. Symmetries, Exact solutions and Conservation Laws, vol. 1, (Boca Raton, CRC Press, 1994).

[32] N.H. Ibragimov and S.V. Khabirov, Contact transformation group classification of nonlinear wave equations, Nonlin. Dyn., 22 (2000), 61-71.

[33] N.H. Ibragimov, M. Torrisi and A. Valenti, Preliminary group classification of equations $v_{t t}=$ $f\left(x, v_{x}\right) v_{x x}+g\left(x, v_{x}\right)$, J. Math. Phys., 32 (1991), 2988-2995. 
[34] N.M. Ivanova, Exact solutions of diffusion-convection equations, Dynamics of PDE, 5 (2) (2008), 139171.

[35] N.M. Ivanova and C. Sophocleous, On the group classification of variable-coefficient nonlinear diffusion-convection equations. J. Comput. Appl. Math., 197 (2) (2006), 322-344.

[36] H. Jia, W.X.X. Zhao and Z. Li, Separation of variables and exact solutions to nonlinear diffusion equations with $x$-dependent convection and absorption, J. Math. Anal. Appl., 339 (2008), 982-995.

[37] O.V. Kaptsov and I.V. Verevkin, Differential constraints and exact solutions of nonlinear diffusion equations, J. Phys. A: Math. Gen., 36 (2003), 1401-1414.

[38] N.A. Kudryashov, On exact solutions of families of Fisher equations, Theor. Math. Phys., 94 (2) (1993), 211-218.

[39] V. Lahno, R. Zhdanov and O. Magda, Group classification and exact solutions of nonlinear wave equations, Acta Appl. Math., 91 (2006), 253-313.

[40] D. Levi and P. Winternitz, Nonclassical symmetry reduction: Example of the Boussinesq equation, $J$. Phys. A, 22 (1989), 2915-2924.

[41] F.-S. Long and S.V. Meleshko, On the complete group classification of the one-dimensional nonlinear Klein-Gordon equation with a delay, Math. Meth. Appl. Sci., 39 (12) (2016), 3255-3270.

[42] S.V. Meleshko and S. Moyo, On the complete group classification of the reaction-diffusion equation with a delay, J. Math. Anal. Appl., 338 (2008), 448-466.

[43] M.C. Nucci and P.A. Clarkson, The nonclassical method is more general than the direct method for symmetry reductions. An example of the Fitzhugh-Nagumo equation, Phys. Lett. A, 164 (1992), 4956.

[44] P.J. Olver, Direct reduction and differential constraints, Proc. Roy. Soc. London, Ser. A, 444 (1994), 509-523.

[45] A. Oron and P. Rosenau, Some symmetries of the nonlinear heat and wave equations, Phys. Lett. A, 118 (1986), 172-176.

[46] A.D. Polyanin, Comparison of the effectiveness of different methods for constructing exact solutions to nonlinear PDEs. Generalizations and new solutions, Mathematics, 7 (5) (2019), 386.

[47] A.D. Polyanin, Construction of exact solutions in implicit form for PDEs: New functional separable solutions of non-linear reaction-diffusion equations with variable coefficients, Int. J. Non-Linear Mech., 111 (2019), 95-105.

[48] A.D. Polyanin, Functional separable solutions of nonlinear convection-diffusion equations with variable coefficients, Commun. Nonlinear Sci. Numer. Simulat., 73 (2019), 379-390.

[49] A.D. Polyanin, Functional separable solutions of nonlinear reaction-diffusion equations with variable coefficients, Appl. Math. Comput., 347 (2019), 282-292.

[50] A.D. Polyanin, Generalized traveling-wave solutions of nonlinear reaction-diffusion equations with delay and variable coefficients, Appl. Math. Letters, 90 (2019), 49-53.

[51] A.D. Polyanin and V.E. Nazaikinskii, Handbook of Linear Partial Differential Equations for Engineers and Scientists, 2nd Edition, (Boca Raton, CRC Press, 2016).

[52] A.D. Polyanin and V.F. Zaitsev, Handbook of Exact Solutions for Ordinary Differential Equations, 2nd Edition, (Boca Raton, Chapman \& Hall/CRC Press, 2003).

[53] A.D. Polyanin and V.F. Zaitsev, Handbook of Nonlinear Equations of Mathematical Physics, (Moscow, Fizmatlit, 2002) (in Russian).

[54] A.D. Polyanin and V.F. Zaitsev, Handbook of Nonlinear Partial Differential Equations, 2nd Edition, (Boca Raton, CRC Press, 2012).

[55] A.D. Polyanin and A.I. Zhurov, Exact separable solutions of delay reaction-diffusion equations and other nonlinear partial functional-differential equations, Commun. Nonlinear Sci. Numer. Simul., 19 (3) (2014), 409-416.

[56] A.D. Polyanin and A.I. Zhurov, Functional constraints method for constructing exact solutions to delay reaction-diffusion equations and more complex nonlinear equations, Commun. Nonlinear Sci. Numer. Simul., 19 (3) (2014), 417-430.

[57] A.D. Polyanin and A.I. Zhurov, Generalized and functional separable solutions to nonlinear delay Klein-Gordon equations, Commun. Nonlinear Sci. Numer. Simul., 19 (8) (2014), 2676-2689. 
[58] A.D. Polyanin and A.I. Zhurov, New generalized and functional separable solutions to non-linear delay reaction-diffusion equations. Int. J. Non-Linear Mech., 59 (2014), 16-22.

[59] A.D. Polyanin and A.I. Zhurov, Nonlinear delay reaction-diffusion equations with varying transfer coefficients: Exact methods and new solutions. Appl. Math. Letters, 37 (2014), 43-48.

[60] R.O. Popovych and N.M. Ivanova, New results on group classification of nonlinear diffusion-convection equations. J. Physics A: Math. General, 37 (2004), 7547-7565.

[61] E. Pucci, Group analysis of the equation $u_{t t}+\lambda u_{x x}=g\left(u, u_{x}\right)$, Riv. Mat. Univ. Parma, 12 (4) (1987), 71-87.

[62] E. Pucci and G. Saccomandi, Evolution equations, invariant surface conditions and functional separation of variables, Physica D, 139 (2000), 28-47.

[63] E. Pucci and M.C. Salvatori, Group properties of a class of semilinear hyperbolic equations, Int. J. Non-Linear Mech., 21 (1986), 147-155.

[64] C. Sophocleous and J.G. Kingston, Cyclic symmetries of one-dimensional non-linear wave equations. Int. J. Non-Linear Mech., 34 (1999), 531-543.

[65] Z. Świerczyński, On the oscillons in the signum-Gordon model, J. Nonlinear Math. Phys., 24 (1) (2017), 20-28.

[66] X.Y. Tang, Z.F. Liang and J.Y. Wang, Nonlocal symmetries and conservation laws of the sinh-Gordon equation, J. Nonlinear Math. Phys., 24 (1) (2017), 93-106.

[67] O.O. Vaneeva, A.G. Johnpillai, R.O. Popovych and C. Sophocleous, Extended group analysis of variable coefficient reaction-diffusion equations with power nonlinearities, J. Math. Anal. Appl., 330 (2) (2007), 1363-1386.

[68] O.O. Vaneeva, R.O. Popovych and C. Sophocleous, Extended group analysis of variable coefficient reaction-diffusion equations with exponential nonlinearities. J. Math. Anal. Appl., 396 (2012), 225242.

[69] O.O. Vaneeva, R.O. Popovych and C. Sophocleous, Group analysis of variable coefficient diffusionconvection equations. I. Enhanced group classification, Lobachevskii J. Mathematics, 31 (2) (2010), $100-122$.

[70] N.N. Yanenko, The compatibility theory and methods for integration of systems of nonlinear partial differential equations, In: Proc. USSR Math. Congress, Nauka, Leningrad, 2 (1964), 613-621 [in Russian].

[71] V.F. Zaitsev and A.D. Polyanin, Exact solutions and transformations of nonlinear heat and wave equations, Doklady Math., 64 (3) (2001), 416-420.

[72] R.Z. Zhdanov, Separation of variables in the non-linear wave equation, J. Phys. A, 27 (1994), L291L297. 